\title{
La transformación
} de las funciones de la asociatividad en una \section{cooperativa de cartoneros: de la representación al trabajo productivo}

The transformation of the functions of a cooperative association of "cartoneros": from the representation to the productive work

Juan Pablo Tagliafico

UBA

ip.tagliafico@gmail.com

Pablo J. Schamber

UNLa y Universidad Nacional de Quilmes Investigador independiente CONICET pjschamber@hotmail.com

\section{Adriana M. García}

Docente-investigadora, Departamento

de Salud Comunitaria, UNLa

Maestranda en Políticas Públicas

y Gobierno, UNLa

adrianamabelgarcia@gmail.com
Fecha de recepción:

13.1.16

Fecha de aceptación:

31.3.16

\section{Resumen}

En las últimas décadas, los recuperadores urbanos de residuos y los procesos de formación de cooperativas han sido objeto de estudio de numerosos análisis. Sin embargo, no son muchos los trabajos que indagan en la heterogeneidad de las cooperativas de recuperadores de la ciudad de Buenos Aires y la especificidad de cada proceso de formación. Como un modo de comenzar a plantear esta cuestión, nos proponemos indagar en el proceso de conformación de una cooperativa en el período 2001-2015, identificando sus particularidades y dando cuenta de los efectos que ha tenido en los cartoneros la participación en esta nueva forma de asociatividad. Nos interesa, además, analizar las transformaciones subjetivas de los propios actores en relación con los procesos asociativos. Las preguntas de las que partimos es para qué se asociaron, para qué formaron cooperativas.

Palabras clave: recuperadores urbanos - cooperativas - trabajo - procesos asociativos - políticas públicas. 


\begin{abstract}
In recent decades, the urban gatherers of recyclables and the processes of formation of cooperatives have been studied in numerous analysis. However, very few attempts have been conducted in order to investigate the heterogeneity of cooperative recyclers of Buenos Aires City and the specificity of each associative process. As a way to to put forward this approach, we propose to investigate the process of building a cooperative of "cartoneros" during 2001-2015, identifying its main characteristics and realizing the effects it has had on their participation in this new form of association. We also analyzed the subjective transformations of the actors in relation to associative processes. The questions we address to answer are: what was the purpose of these individuals to join an association? What was their purpose to build a cooperatives?
\end{abstract}

Key words: urban gatherers - cooperatives - labor - associative process - public policies.

\title{
Introducción ${ }^{1}$
}

La actividad conocida como "cirujeo", recolección informal de residuos o, en las últimas décadas, "cartoneo", tiene una larga historia en toda el Área Metropolitana de Buenos Aires (AMBA) en general y en la Ciudad de Buenos Aires (CABA) en particular. Diversos estudios, desde perspectivas y con objetivos diferentes han abordado la historia de esta problemática, ligándola con los distintos modos de gestión de los residuos (Paiva, 2005; Perelman, 2008; Schamber y Suárez, 2006; entre otros). Al mismo tiempo, junto con las transformaciones que se desencadenaron en las últimas décadas en los sistemas de recolección en la CABA, se produjo una amplia literatura sobre el tema (Fajn, 2002; Escliar et al, 2007; Gorbán, 2006; Reynals, 2002; Paiva, 2004, 2013; Schamber y Suárez, 2012; Villanova, 2014).

Los cartoneros y los procesos de formación de cooperativas de trabajo han sido elementos muy destacados en los distintos análisis. Sin embargo, no son muchos los trabajos que indaguen en la heterogeneidad de las cooperativas de recuperadores de la CABA y la especificidad que caracteriza a cada proceso de formación. Partiendo de este punto nos propusimos indagar en el proceso de formación de una cooperativa, identificando sus 
particularidades y dando cuenta de los efectos que ha tenido en el colectivo de los recuperadores urbanos la participación en esta nueva forma de asociatividad. Especialmente nos interesa analizar las transformaciones subjetivas de los propios actores en relación con los procesos asociativos. La pregunta de la que partimos será, entonces, para qué se asociaron y para qué formaron cooperativas.

Para esto, tomaremos el caso de la Cooperativa de Recuperadores Urbanos del Oeste (CRUO), una de las cooperativas de recuperadores de residuos sólidos urbanos (RSU) secos más importante de la CABA. En la actualidad nuclea aproximadamente a 1.500 trabajadores, representando así la segunda cooperativa más grande del sector en toda la ciudad, en lo que a número de trabajadores respecta. ${ }^{2} \mathrm{Su}$ área de trabajo está constituida entre los barrios de Liniers y Once, siendo esta última zona la de mayor relevancia debido a la cantidad de material reciclable que se genera producto de su intensa actividad comercial. Es una de las 13 cooperativas que participa del sistema de recolección de RSU secos de la CABA, tras haber participado de la licitación llevada a cabo por el GCBA (Schamber, 2012) y firmado, finalmente, el contrato el 2 de enero del 2013. Este sistema de recolección se encuentra, en la actualidad, en proceso de constante evaluación y cambio y esto también se observa en la cooperativa. En este artículo ahondaremos, primeramente, en la situación de los recuperadores urbanos en la CABA, indagando, específicamente, en el proceso que ha atravesado el heterogéneo conjunto de cartoneros de la ciudad para llegar a la conformación en cooperativas y las transformaciones de la legislación sobre el tema que se han impulsado. En segundo lugar, introduciremos el caso de la CRUO, analizando las diferentes etapas que ha atravesado el conjunto de cartoneros y los distintos modos en que se han organizado. Para concluir, realizaremos un análisis de los procesos asociativos para lograr esbozar algunas respuestas a nuestras preguntas iniciales. ${ }^{3}$

\section{La situación en CABA}

Con la creación de la Coordinación Ecológica Área Metropolitana Sociedad del Estado (CEAMSE), desde mediados de la década de 1970 se privilegió el entierro indiscriminado de residuos a partir de la implementación del método de relleno sanitario (Perelman, 2008: 128; Schamber y Suárez, 2002: 4). Como efecto de las políticas macroeconómicas impulsadas desde entonces y profundizadas durante la década de 1990 se transformó la estructura económica argentina y su mercado de trabajo, lo que generó el empobrecimiento de la clase trabajadora y el aumento del desempleo. La conexión entre ambos fenómenos se evidencia en la opinión pública (Schamber y Suárez, 2012: 108) luego de la crisis económica, social y política de fines de 2001, cuando la "explosión" del número de cartoneros (denominados como "nuevos cirujas" o "nuevos cartoneros" en Paiva, 2013: 152 y Perelman y Boy, 2010: 403, entre otros) desnudó la inexistencia de políticas públicas que promovieran el reciclaje. ${ }^{4}$ 
La amplia visibilidad que adquirió el fenómeno produjo una respuesta desde la Legislatura de la CABA. El 12 de diciembre de 2002 se sancionó la ley 992, publicada en el Boletín Oficial (BOCBA) No 1619 el 29 de enero de 2003. ${ }^{5}$ En su artículo 2 2o "el Poder Ejecutivo incorpora a los recuperadores de residuos reciclables a la recolección diferenciada en el servicio de higiene urbana vigente", y en su artículo $3^{\circ}$ dispone una serie de cinco objetivos, entre los que deja "sin efecto, como disposición final, el entierro indiscriminado de los residuos en los rellenos sanitarios". En este sentido, su artículo $7^{\circ}$, deroga el artículo $6^{\circ}$ de la Ordenanza $N^{\circ} 33.581$, el cual prohibía, desde junio de 1977, la manipulación de residuos, es decir, el cirujeo o cartoneo. A partir de esta legislación "los recuperadores podían hacerse cargo del retiro de productos reciclables en los denominados 'grandes generadores', es decir, supermercados, oficinas estatales, hoteles, etc.” (Villanova, 2014: 73).

El artículo $4^{\circ}$ crea el Registro Único Obligatorio Permanente de Recuperadores de Materiales Reciclables (RUR), el cual debía inscribir a los recuperadores, entregándoles credenciales, vestimenta para el trabajo y guantes. A su vez, el artículo $5^{\circ}$ dispone la creación de un "Registro Permanente de Cooperativas y Pequeñas y Medianas Empresas relacionadas con la actividad". Debemos destacar, además, que la nueva denominación como "recuperadores" resulta significativa en términos subjetivos ya que, según Paiva, "se intentó valorizar la tarea al resignificar la antigua figura del ciruja por la del 'recuperador de residuos"” (Paiva, 2013: 154).

La ley 992 fue reglamentada a través del decreto n 622 en mayo de $2003 .{ }^{6}$ En el artículo $1^{\circ}$ de su Anexo, se crea el Programa de Recuperadores Urbanos y Reciclado de Residuos Sólidos Urbanos (PRU), como organismo competente para tratar la problemática, legislándose sobre su composición y funcionamiento. Sin embargo, "a pesar de haber sido incorporados a la recolección diferenciada, aún no existía una infraestructura por la cual los cartoneros pudieran recolectar esos materiales" (Villanova, 2014: 73), por lo que esta política se mostró considerablemente limitada. En palabras de otros autores:

De todos modos, la incorporación de los recuperadores urbanos a la recolección diferenciada del servicio de higiene urbana, en los hechos resultó meramente relegada a la inscripción de los mismos en el RUR. Se podría decir que la inscripción en el RUR y la consecuente entrega de credenciales y vestimenta de trabajo fue la manera en la que se incorporó a los recuperadores al servicio público de higiene urbana. Aun así, también es importante aclarar que la compra de la vestimenta mencionada mediante el procedimiento y los tiempos propios de la administración pública impidió que todos los inscriptos pudieran obtener- 
la en el momento del registro, y en muchos casos ni siquiera al cabo de varios meses después. Lejos quedó el PRU en ese entonces de convertirse en la oficina del gobierno con una real injerencia en el diseño y la ejecución de una política favorable al reciclaje socialmente incluyente (Schamber y Suárez, 2012: 111).

Entre 2003 y 2005 distintas problemáticas sociales y urbanas atravesaron la cuestión de los cartoneros y del sistema de recolección de residuos. Entre las principales encontramos:"el trabajo infantil, el acceso a la ciudad desde el conurbano bonaerense en camiones no habilitados, el conflicto entre depósitos de acopio y vecinos, la clasificación de materiales en la vía pública" (Schamber y Suárez, 2012: 113).

En noviembre de 2005 se sancionó la ley 1.854, conocida como "Ley de Basura Cero".7 En su artículo 10 se propone "promover la participación de cooperativas y organizaciones no gubernamentales en la recolección y reciclado de los residuos". A su vez, el artículo 44 prescribe que la Ciudad adoptará "las medidas necesarias para establecer líneas de crédito y subsidios destinados a aquellas cooperativas de recuperadores urbanos inscriptas en el Registro Permanente de Cooperativas y de Pequeñas y Medianas Empresas (REPyME)", con el fin de adquirir bienes de capital para su actividad. A través de esta ley se propone también la construcción de centros de selección de residuos sólidos urbanos secos, también denominados Centros Verdes: "El eje central de la normativa fue la creación de plantas de clasificación con el propósito de disminuir la basura destinada a los rellenos sanitarios debido a que éstos se encontraban a punto de colapsar" (Villanova, 2014: 73). En este sentido, en el artículo 14 se dispone la obligación de los grandes generadores de residuos sólidos urbanos secos de "realizar la separación en origen y adoptar las medidas tendientes a disminuir la cantidad de residuos sólidos urbanos que genere". Al mismo tiempo se establece la incorporación de contenedores para los residuos sólidos urbanos secos y su recolección diferenciada de los residuos húmedos. A pesar de esto,

La normativa priorizaba la inclusión de cartoneros en el proceso de recolección y transporte del reciclado. Sin embargo, los requisitos de cada vehículo estaban por fuera del alcance de los cartoneros. A su vez, la ley indicaba que aquellos prestadores o quienes aspirasen a participar de dicho servicio de transporte debian presentar, entre otras obligaciones, una póliza de seguros que cubriera daños, poseer vehículos con sistemas de comunicación, garantizar el tratamiento correspondiente de los efluentes generados por la actividad; garantizar la limpieza de contenedores y recipientes de residuos sólidos urbanos en forma periódica 
para evitar el escape de polvos, desperdicios y/o generación de microbios y bacterias; $y$, capacitar al personal afectado al transporte y recolección diferenciada. Dada la imposibilidad por parte de los cartoneros de efectuar esta tarea, la recolección quedó a cargo de las empresas privadas de recolección de residuos

(Villanova, 2014: 74).

Durante 2006, en el marco de la ley 1.854, el GCBA obligó a los grandes generadores a separar los materiales reciclables de los residuos, a las empresas privadas de recolección a colocar contenedores en las calles para la separación del material y, al mismo tiempo, comenzó la construcción y puesta en funcionamiento de los Centros Verdes. ${ }^{9}$ Sin embargo, a partir de 2008, una nueva administración a cargo del GCBA tomó la decisión de modificar nuevamente el sistema. Esta modificación puede ser entendida a partir de dos elementos importantes: "la deficiente prestación del servicio por parte de las empresas privadas y la suspensión de los 'trenes cartoneros' de TBA (en 2007)"10 (Schamber y Suárez, 2012: 115). Así, el 7 de julio de 2008 el GCBA firmó con diversas cooperativas de cartoneros un acuerdo para llevar adelante una nueva política de reciclado: en el mismo, el GCBA se comprometió a dejar en concesión como Servicio de Higiene Urbana sólo los residuos húmedos o no reciclables y a incorporar a los recuperadores urbanos como protagonistas del sistema de recolección de RSU secos, brindando un servicio de transporte para los cartoneros y para sus carros, y proveyendo la logística y el transporte para el traslado desde los grandes generadores de residuos hacia los Centros Verdes gestionados por los cartoneros (Schamber y Suárez, 2012: 118). Entre el 2008 y el 2010:

Los acuerdos logrados entre las organizaciones de los recuperadores y el Gobierno se fueron extendiendo en términos de entrega de subsidios, asignación de recorridos de recolección en grandes generadores mediante el otorgamiento de camiones en comodato y/o la cesión de Centros Verdes para su gestión, y alcanzaron a otros grupos de cartoneros (Schamber y Suárez, 2012: 119).

Esta etapa que comienza en 2008 significó, a su vez, un proceso de discusión y de disputa en torno al nuevo sistema de recolección de residuos, donde la intervención de las cooperativas y su posicionamiento frente a otros actores, como el GCBA, la Legislatura o algunas ONG será un elemento importante (Schamber y Suárez, 2012; Villanova, 2014). Este proceso habría de culminar en abril 2010 con la presentación de los pliegos (Expediente $N^{o} 350.165 / 2010$ ) para la licitación pública de la contratación del servicio de recolección de residuos húmedos y con un concurso público para la prestación del servicio de recolección de RSU secos. De esta forma, se ponía en funcionamiento un nuevo sistema de recolección, a través del cual el Estado local seguiría gestionando la recolección de residuos húmedos a través de la contratación de empresas privadas y, al mismo tiempo, negociaría con las cooperativas de recuperadores las condiciones para la recolección de residuos reciclables. 
Como principales características del sistema de recolección de RSU secos, debemos mencionar que las cooperativas de recuperadores urbanos se hacen cargo de los Centros Verdes y de la recolección de los RSU secos, permitiendo el GCBA que las mismas puedan hacer uso de las ventas del material recogido. Al mismo tiempo, se concursan las distintas zonas de trabajo en la ciudad, debiendo las cooperativas incluir a los cartoneros individuales que se encuentren trabajando en las zonas asignadas. El GCBA se compromete también a la implementación de distintos programas que garanticen los derechos de los trabajadores y la correcta implementación del servicio (erradicación del trabajo infantil, un programa de inclusión social, el manejo de la logística en el proceso de trabajo, incentivos económicos mensuales). Finalmente, luego de una audiencia pública en julio de 2010, se firmaron los contratos el 29 de marzo del 2011 entre el GCBA, las empresas privadas y las cooperativas de recuperadores urbanos, para la prestación de los servicios de recolección. ${ }^{11}$ El plazo fue de 4 años, con la posibilidad de prorrogarse por un año más. Postergados casi dos años, los contratos del servicio público de recolección de RSU secos entre el GCBA y 13 cooperativas de recuperadores urbanos fueron vueltos a firmar el 2 de enero de 2013. ${ }^{12}$

En el marco de este proceso (ver Cuadro I), la actividad de los cartoneros, el proceso y las condiciones de trabajo se han ido modificando. Al mismo tiempo, su trayectoria estuvo caracterizada por el surgimiento de numerosas cooperativas de trabajo que fueron estrechando diferentes lazos con el GCBA y el estado local. Como un modo de dar cuenta, aunque parcialmente, de este proceso, seleccionamos el caso de una de las cooperativas de la CABA, la Cooperativa de Recuperadores Urbanos del Oeste.

\section{La Cooperativa de Recuperadores Urbanos del Oeste (CRUO)}

\subsection{La conformación como cooperativa (2001-2008)}

Una gran parte de los actuales integrantes de la CRUO provienen del oeste del Gran Buenos Aires (GBA). Existe dentro de la cooperativa un grupo histórico de recuperadores urbanos que se trasladaban desde localidades del oeste del GBA (Moreno, Paso del Rey, Merlo, Padua, etc.) hacia la CABA para recolectar materiales reciclables. Esos viajes en los trenes cartoneros de la Línea Sarmiento (de Moreno a Once), desde el año 2004, significaron las primeras experiencias organizativas entre estos recuperadores urbanos. De acuerdo a Lidia Chedde, secretaria de la cooperativa y coordinadora general de un sector del Centro Verde Yerbal, y Valeria Díaz, una de las fundadoras y hoy vicepresidente de la cooperativa, los primeros lazos sociales y el surgimiento de las formas asociativas entre los cartoneros del oeste del GBA se fueron construyendo a partir de la experiencia común que atravesaban al viajar en el tren hacia la CABA:

Nos conocíamos todos por el tren cartonero... De Once a Moreno... yo soy de Merlo. Y ahí nos fuimos conociendo 
todos. Y después de ahí quedé de delegada... Me eligieron por buena compañera

(Lidia Chedde, secretaria y coordinadora de la CRUO, entrevista de los autores, 08/10/2014).

Nos fuimos conociendo en la lucha por el tren, para que nos den el tren cartonero $y$ así nos fuimos organizando (Valeria Díaz, vicepresidenta de la CRUO, entrevista de los autores, 06/01/2016).

Luego, en el tren cartonero, se fueron consolidando esos primeros lazos sociales y esas primeras formas de asociación entre los recuperadores urbanos. ${ }^{13}$

Se esbozaron así las primeras formas de organizar el trabajo en conjunto: a través de la organización en los distintos vagones se fueron distribuyendo los distintos puntos de bajada en la CABA (desde Liniers hasta Once) de acuerdo a los puntos de subida al tren (Moreno, Paso del Rey, Merlo, Padua, etc.). Esta trayectoria común que presenta un grupo histórico dentro de la cooperativa es una característica distintiva y muy presente entre los integrantes de la CRUO:

Lo del tren cartonero estuvo bien porque en el otro siempre teníamos problemas con los que viajaban... molestábamos y nos discriminaban. En el tren cartonero viajábamos mejor $y$ ahí nos fuimos conociendo

(Valeria Díaz, vicepresidenta de la CRUO, entrevista de los autores, 06/01/2016).

Así, al momento de sancionarse la ley 1.854 en 2005, ya existían lazos sociales que estrechaban las relaciones entre estos cartoneros y los mantenían organizados. La ley significó un fuerte impulso para el proceso de organización del trabajo entre los recuperadores urbanos. A partir de ese momento los cartoneros de la CABA, a través de la conformación de cooperativas, pasaban a constituir parte formal del sistema de recolección de RSU secos y se abría un proceso de disputa con el GCBA en torno al rol que debían ocupar en ese sistema.

El año 2008, como vimos, estuvo marcado principalmente por la suspensión de los trenes cartoneros, el inicio del servicio de transporte brindado por el Estado local a través de camiones y la deficiente prestación del servicio de recolección por parte de empresas privadas, hechos que trajeron como consecuencia la apertura del proceso de licitación del servicio. La suspensión de los trenes afectó considerablemente a los cartoneros de la actual CRUO e impulsó un proceso de lucha para obtener un servicio que resolviera las necesidades de traslado del conjunto de los cartoneros: 
Cuando nos sacan el tren cartonero, acordamos que nos pongan los camiones. Nos plantamos a la mañana y hasta la noche no nos íbamos sin los camiones... Y a las nueve de la noche nos pusieron los camiones

(Valeria Díaz, entrevista de los autores,06/01/2016).

Junto a estos elementos, tras el proceso de licitación del servicio, se comenzó a otorgar incentivos "por capacitación relativa a la recolección y reciclado", un subsidio de 200 pesos en ese momento (Villanova, 2012: 109). ${ }^{14}$ Más allá de si constituyeron "mecanismos de cooptación por parte del gobierno porteño" (Villanova, 2012:108) o de las distintas intenciones de los sujetos, debemos señalar que, en términos concretos, tanto los nuevos camiones como los incentivos han sido elementos importantes para impulsar el desarrollo del cooperativismo entre los cartoneros. Tras la sanción de la ley 992, el decreto 622 y la ley 1.854 , lo que marcará el proceso de licitación iniciado en 2008, junto con los nuevos camiones, los incentivos y la implementación de este nuevo sistema de recolección de residuos es la profundización de un proceso de institucionalización a través de distintos organismos del Estado local creados para tratar la problemática.

Por otro lado, las alianzas que distintos grupos de cartoneros estrecharon con diferentes organizaciones se modificaron en el tiempo y son un elemento importante para comprender el proceso de asociación. En el caso de la CRUO, existen importantes lazos entre la cooperativa y distintos agentes ligados a la administración pública y, a su vez, a la militancia sindical en la Asociación Trabajadores del Estado (ATE). Son estos agentes quienes desde el 2008 han brindado nuevas herramientas en el proceso de organización en cooperativas como un modo de establecerse como interlocutores válidos frente al GCBA y al Estado local. Por su conocimiento técnico acerca de los procesos burocráticos y por su compromiso político ligado a los cartoneros, estos agentes jugaron un rol muy importante a la hora de articular las relaciones entre la cooperativa y el Estado local. ${ }^{15}$

Como vemos, durante estos años, se vivió un proceso de mayor organización entre los cartoneros ("recuperadores urbanos" desde el 2005), surgiendo así nuevas formas asociativas. Las novedosas asociaciones se organizaban, principalmente, en torno a dos funciones. Por un lado, les permitía establecerse como interlocutores válidos frente al GCBA y al Estado local, centralizando sus reclamos y reivindicaciones. Por el otro, planteaban nuevas formas de organizar el trabajo. En relación con la primera de estas funciones, las nuevas cooperativas les permitieron a los recuperadores urbanos presentarse ante el GCBA como parte de la solución para la gestión de los residuos y no como un problema, como interlocutores válidos con los cuales era necesario discutir e integrar en el re-diseño de las políticas públicas en torno a la gestión de RSU secos. En este sentido, durante estos años lograron abandonar una posición relativamente pasiva respecto al diseño de políticas públicas en relación a la gestión de residuos, pasando a discutir concretamente el modo de 
funcionamiento del propio sistema. Con respecto a la organización del trabajo, la formación de la CRUO, en particular, estuvo mediada por el acceso al transporte y al cobro de los incentivos. Sin embargo, la formación de la cooperativa no modificó sustancialmente las condiciones de trabajo. Los camiones y colectivos que comenzaron a brindar el transporte pueden haber encontrado cierta resistencia entre algunos integrantes de la cooperativa, pero seguían manteniendo la misma dinámica de trabajo. Los incentivos, por un lado, significaron un considerable aporte económico para quienes lo percibían, aunque por otro lado, muchos trabajadores debieron esperar para comenzar a percibirlo. ${ }^{16} \mathrm{La}$ dinámica de trabajo continuaba siendo la misma, teniendo que trasladarse desde sus hogares hacia la CABA con sus carros y vendiendo su material de forma individual a los galponeros. De esta forma, hasta el 2008 el funcionamiento como cooperativa se limitaba a una cuestión representativa frente al GCBA y al Estado local, así como también frente a organizaciones de la sociedad civil, empresas, vecinos, etc.

\subsection{La cooperativa en un nuevo modelo de recolección (2008-2013)}

De acuerdo a lo señalado, durante 2008 inició el proceso de licitación del servicio. En el caso de la CRUO, ese año estuvo marcado por la fuerte exigencia para que se le asignase un Centro Verde, tal como lo establecía la ley 1.854. Tras el proceso de quiebra de la Cooperativa Ecológica de Recicladores del Bajo Flores (CERBAF), los recuperadores urbanos de la CRUO decidieron tomar el terreno del entonces llamado Centro Verde del Bajo Flores, ubicado en Villa Soldati. Luego de una disputa con el GCBA, la CRUO logró que se le asignara el terreno, iniciándose su actividad en lo que hoy se conoce como CentroVerdeVarela. ${ }^{17}$

Inicialmente, este CentroVerde no modificó la dinámica de trabajo de los recuperadores urbanos de la cooperativa. La distancia entre el Centro Verde Varela, en Villa Soldati, y la zona de trabajo, entre Once y Liniers (ver Mapa I), significó un obstáculo para mejorar el proceso de producción y las condiciones de trabajo de los recuperadores urbanos. Estos seguían trasladándose con los carros desde sus hogares hacia la CABA, subiéndolos y bajándolos de los camiones. Continuaban, a su vez, regresando con el material para vender lo recolectado de forma individual o en pequeñas asociaciones a galponeros del GBA. Mientras tanto, la función del Centro Verde Varela se limitaba a la recepción del material de algunos grandes generadores de RSU secos de la CABA.

Durante el periodo 2008-2013 al que se refiere este acápite, se ha desarrollado en la CRUO un proceso en el que, atravesando diversas manifestaciones e incluyendo acciones de protesta, ha aumentado constantemente el número de cooperativistas y el número de recuperadores urbanos con acceso a ropa de trabajo, guantes, transporte e incentivos. El proceso vivido en la CRUO tiene correlación con lo que ocurre en el resto de las cooperativas del sector. 
En términos generales, las acciones del período entre 2009 y 2011 se caracterizan fundamentalmente por la obtención de más subsidios, su renovación, incremento de los cupos y aumento de su monto. En su abrumadora mayoría, las manifestaciones fueron convocadas por las cooperativas cartoneras, aunque de manera fragmentada (Villanova, 2012: 109).

Esta ampliación de mejoras concretas trajo como consecuencia, a su vez, otro proceso que podemos denominar de reconocimiento de la propia actividad como un trabajo y de su propia situación como trabajadores. Los cambios concretos que hemos mencionado han producido en la propia subjetividad de los cooperativistas, por un lado, la percepción de que su actividad es un trabajo. Mendoza, coordinador de un sector del Centro Verde Yerbal, nos comentaba acerca del impacto que tuvo en la propia actividad la implementación de los incentivos y el "tomar lista" diariamente:

Todos tienen que tener presente, porque sino después tienen descuento en el incentivo... Acá es estricto. No porque seas cartonero vos venís cuando se te canta... No, no es así. Primero hacían así hasta que hubo un incentivo. Cuando hubo un incentivo, bueno... Entonces, ellos tienen que dar el presente ahora... después de las seis de la tarde tienen que dar el presente acá, y después va y le toman los del Gobierno allá también... Antes del incentivo cada uno venía cuando quería, como quería. Sí, señor. Exactamente. Así era. Ahora no. Ahora es diferente. Ahora tenés que venir todos los días

(Mendoza, coordinador en el Centro Verde Yerbal de la CRUO, entrevista de los autores, 08/10/2014).

Por otro lado, los entrevistados nos han relatado también los cambios percibidos en los modos en que otros sectores de la sociedad los interpelan. Ambos elementos conforman una situación que se contrapone a la de principios de siglo donde la propia actividad podía ser vivida con vergüenza, desprecio, desconfianza, considerada un delito, etc. Como relatan Perelman y Boy, a principios de este siglo, los “cirujas” vivían

en un constante juego de equilibrio entre el rechazo, la vergüenza y la utilización del uniforme de pobreza como forma de acceder a mercaderías tales como ropa, alimentos, materiales en desuso, entre otros

(Perelman y Boy, 2010: 412). 
Al comenzar su actividad, principalmente los "nuevos cartoneros" vivían en una permanente tensión "entre esconder la diferencia y dar cuenta de ella", cargando con el estigma de ser reconocidos como cartoneros (íd. loc.cit). Este estigma se encuentra por lo menos puesto en cuestión, en la actualidad. No todos se avergüenzan de su trabajo o se sienten mirados como delincuentes o vagos. Por el contrario, muchos perciben que son reconocidos como trabajadores, se enorgullecen del lugar que ocupan hoy y de las condiciones de trabajo alcanzadas. Al mismo tiempo, esto es percibido, en la mayoría de los casos, como una consecuencia de la organización y la lucha que ellos mismos han llevado adelante para alcanzar ese reconocimiento. Esto se acompaña entonces, en muchos casos, con el orgullo de atravesar una experiencia no sólo de trabajo, sino también militante. Los recuperadores urbanos suelen hacer alusión a que todos los derechos obtenidos o consolidados durante estos años han sido logros producto de las luchas llevadas adelante por las distintas organizaciones: movilizaciones, cortes de calle, tomas de terrenos, etc.

Hoy estamos mejor... Logramos muchas cosas con la cooperativa, pero todo fue una lucha... Marchas, cortes de calle, ministerios... Y así conseguimos este predio, la obra social, las credenciales y todo

(Valeria Díaz, vicepresidenta de la CRUO, entrevista de los autores, 06/01/2016).

En esta primera etapa se ha producido, entonces, la institucionalización de los lazos entre los recuperadores urbanos a través de los procesos de organización en cooperativas. Al mismo tiempo, estas nuevas asociaciones permitieron ampliar el acceso a mejoras concretas en relación con aquellos cartoneros no asociados. A través de la organización se articularon también reclamos y acciones de protesta, a la vez que se institucionalizaron las relaciones con el estado local y el GCBA. Sin embargo, en esta etapa, la organización como cooperativa, en el caso de la CRUO, mostró sus limitaciones para organizar el proceso productivo. La introducción del Centro Verde Varela no logró modificar la actividad laboral de la gran mayoría de los cartoneros, quienes seguían realizando la misma actividad de recolección y comercialización de los productos. Los recuperadores urbanos partían de sus hogares con sus carros, subían a los camiones y eran trasladados a la CABA para realizar allí sus rutas. Una vez finalizado el recorrido, eran trasladados nuevamente hacia sus hogares donde acopiaban parte del material, clasificándolo y vendiéndolo a los galponeros de su zona. El Centro Verde Varela se limitaba a recibir el material de los grandes generadores asignados a la cooperativa, clasificándolo y vendiéndolo.

De esta forma, la cooperativa representaba en esta etapa una forma de acceder a los derechos conquistados $\mathrm{y}$, a su vez, de presentarse como interlocutores válidos frente al GCBA. Al mismo tiempo, era una forma de concentrar los reclamos, mantener los lazos 
entre los recuperadores urbanos y organizar la lucha frente al GCBA, funcionando como institución necesaria para la intercomunicación entre los recuperadores urbanos y como representante de sus intereses. Por estos motivos es que creemos pertinente definir a la cooperativa, en estos momentos, por su predominante función representativa que prevalece en esta etapa y que cumple tanto a los ojos de los recuperadores urbanos como del GCBA.

\subsection{Las trasformaciones de la cooperativa: de la representación al trabajo productivo (2013-2015)}

A partir de la firma de los contratos entre las 13 cooperativas y el GCBA el 2 de enero 2013, habrían de producirse cambios importantes en las condiciones de trabajo de los recuperadores urbanos. Los trabajadores de la actividad fueron incorporados por la CABA al sistema previsional como monotributistas, pasando a contar con un seguro por accidentes personales durante su jornada de trabajo, junto con una obra social, para ellos y sus familias. Esto significó, entonces, una modificación importante en términos de seguridad social y salud pública. Al mismo tiempo, al formalizarse las relaciones entre las cooperativas y el estado local, algunos empleados estatales, ligados al Ministerio de Ambiente y Espacio Público de la CABA, comenzaron a desempeñar diversas actividades con las cooperativas, desde "pasar lista" a los recuperadores urbanos de la cooperativa, el acompañamiento y evaluación de la jornada de trabajo, hasta cuestiones de logística y planificación.

Ese mismo año la CRUO llevó a cabo la toma de unos terrenos linderos a las vías del ferrocarril de la línea Sarmiento, ubicados en la calle Yerbal 1500, en el barrio de Caballito. Igual que en el caso de los terrenos de Villa Soldati, el GCBA asignó el predio a la CRUO para constituir un nuevo centro de tratamiento, el Centro Verde Yerbal. Ubicado en el centro de la zona de trabajo de la cooperativa, este Centro Verde ya no presenta el obstáculo de la lejanía como el Centro Verde Varela (ver Mapa I). Con ambos en funcionamiento la CRUO transformó su dinámica de trabajo.

Por un lado, los recuperadores urbanos modificaron sus condiciones de trabajo. Salen de sus hogares sin los carros hacia el Centro Verde Yerbal y desde allí parten con ellos hacia sus zonas de trabajo. Al finalizar la jornada regresan al Centro Verde Yerbal, donde realizan la separación y clasificación del material recolectado para la posterior venta del mismo. El material recolectado por los recuperadores urbanos es vendido por ellos mismos a la CRUO, la cual desde 2013 es la encargada de adquirir el material de todos los trabajadores. De esta forma la cooperativa está en condiciones de comercializar mayor cantidad de material, intentando de esta forma vender a niveles industriales que antes resultaban más allá de sus posibilidades. Anteriormente a la firma de los contratos el mercado formal le estaba negado a las distintas cooperativas ya que no sólo no manejaban las cantidades de materiales suficientes para la venta directa a grandes industrias, sino que tampoco poseían los requisitos formales (facturación de ventas, código único 
de identificación tributaria, etc.) para el registro de sus ventas. Con la centralización de las compras, la CRUO alcanzó un margen monetario mayor para ofrecer mejores precios a sus socios. Ello no obstante, siguen manteniéndose en la actualidad relaciones comerciales con intermediarios. Si bien la mayor cantidad de material manejado por la CRUO junto con la incorporación de los requisitos formales mencionados, ha permitido a la cooperativa intentar establecer relaciones comerciales con el sector industrial, han surgido nuevos obstáculos que han atentado contra la solidez de esos vínculos de forma estable o duradera, manteniéndose el recurso a los comerciantes intermediarios. ${ }^{19}$

Aunque estos factores no han permitido hasta ahora el acceso a niveles industriales, la organización ha comenzado a manejar una gran cantidad de material al comprárselo a los cartoneros, obteniendo así una considerable capacidad de negociación y logrando mejores acuerdos con los galponeros o intermediarios a los que vende. De este modo, la ganancia que anteriormente quedaba en quienes poseían la capacidad de acopiar grandes cantidades de material se traslada hoy, en distintas proporciones, a la cooperativa por un lado, y a los recuperadores urbanos por otro. En algunos materiales, en ocasiones, el valor ofertado por kilo de material por parte de la cooperativa es casi $300 \%$ mayor que el de los galponeros del GBA. ${ }^{20}$

Por otro lado, la puesta en funcionamiento del Centro Verde Yerbal ha significado para los recuperadores urbanos la incorporación a su actividad de un lugar de trabajo. A diferencia de lo que sucedía antes del 2013, los recuperadores urbanos comienzan a realizar parte de su trabajo fuera de la calle. Simbólicamente esto nos parece significativo en términos de la imagen que logran construir los recuperadores urbanos de ellos mismos. Es decir, en términos de construcción o, en algunos casos, re-construcción de su propia identidad como trabajadores. Lo que queremos resaltar en este sentido es que, sumado a la obligatoriedad de asistir a trabajar todos los días para poder cobrar el incentivo económico del GCBA, desde el 2013 los recuperadores urbanos comienzan a percibir que asisten a su lugar de trabajo. Se construye en los recuperadores urbanos, a partir de esta novedosa situación, su identidad como trabajadores. Esta identidad se pone en juego frente al GCBA, frente al resto de la sociedad y, al mismo tiempo, entre los propios recuperadores urbanos. Éstos se posicionan como trabajadores percibiéndose como sujetos de derechos que deben ser reconocidos por el Estado de la Ciudad, por el GCBA, por el sector empresario y por el resto de la sociedad. La incorporación al sistema formal de gestión de los RSU secos ha transformado a los recuperadores urbanos en interlocutores válidos frente a otros actores sociales que conforman la ciudad. Frente a una antigua actitud de rechazo o de desconfianza, los recuperadores urbanos contraponen una situación actual donde son vistos y tratados hoy en día con aceptación y como trabajadores que ejercen un trabajo fundamental para la ciudad. En lo que podemos definir como una profundización de los cambios en las subjetividades que vienen llevándose a cabo desde la conformación en cooperativas, los recuperadores urbanos conviven en una constante tensión donde parecen dejar atrás sentimientos de vergüenza o rechazo por la tarea que realizan y comienzan a concebirse 
como trabajadores que realizan una tarea no sólo importante sino necesaria para la ciudad. Yo siempre lo sentí como un trabajo. Capaz otros de otros grupos no, pero para los míos (por los de su grupo) siempre fue un trabajo... Para los de afuera sí, antes éramos delincuentes... o por ejemplo, los del shopping nos querían vender el material. Ahora no... Los vecinos nos conocen, nos llaman, nos acercan el material

(Valeria Díaz, entrevista de los autores, 06/01/2016).

Al mismo tiempo, dejan de ser estigmatizados por una parte de la sociedad, pero no logran dejar de ser criminalizados, principalmente por las fuerzas policiales:

Lo de la credencial estuvo bien, porque sino seguíamos teniendo problemas con la policía. Yo la pido sobre todo por los más pibes que cuando vienen en tren la policía los jode por cómo se visten

(Valeria Díaz, vicepresidenta de la CRUO, entrevista de los autores, 06/01/2016).

Sin embargo, más allá de que existe una consolidación de la identidad como trabajadores y un proceso de desprendimiento de ciertos estigmas, asimilable con procesos descriptos por otros autores (Dimarco, 2007), estos análisis deben ser contrastados con otras perspectivas que ponen en cuestión la linealidad de las relaciones entre vergüenza y orgullo (Vergara, 2009). La cuestión de las subjetividades parecería requerir análisis más profundos que logren destacar la compleja trama en que se ponen en relación la autoestima y los estigmas con las problemáticas de los residuos, la criminalidad y la pobreza.

Junto a estas transformaciones se ha visto modificada la función del Centro Verde Varela. Con el aumento considerable del material manejado por la cooperativa, tras la compra a los recuperadores urbanos, los materiales reciclables son enviados desde Yerbal a Varela, donde no sólo se reciben los materiales de los grandes generadores de RSU secos de la CABA, sino también, desde 2013, los papeles, cartones y plásticos que los recuperadores urbanos le venden a la CRUO; los papeles y los cartones son enfardados en Varela para luego ser comercializados desde allí.

De esta forma, desde 2013 la CRUO ya no es sólo una forma de relacionarse con el GCBA o de representar los intereses de los recuperadores urbanos, ni tampoco se limita a cumplir la función de mantener los lazos entre los trabajadores. En la actualidad la cooperativa establece un precio de compra del material de los recuperadores urbanos, organiza los grupos de trabajo, administra gran cantidad de materiales reciclables, enfarda papeles y cartones, administra la venta del material, negocia con medianas y grandes industrias, 
busca y negocia con grandes generadores de material reciclable para recibir sus reciclables, etc. La cooperativa juega un rol fundamental en la comercialización, en el circuito de los materiales reciclables y en todo el proceso productivo, participando activamente en la organización del trabajo; en ella no sólo se discute acerca del proceso de trabajo y la organización que adopta, sino que se proponen modificaciones al sistema establecido por el GCBA. La presencia de empleados estatales dentro de la cooperativa constituye un eje de tensiones en las que se juegan roles de patrón-empleado, conocimiento técnico-conocimiento práctico, etc., pero al mismo tiempo ha significado la apertura de canales de comunicación más fluidos en los que se pone en discusión el propio sistema de gestión de los residuos. Es por todo esto que creemos que, desde la incorporación del Centro Verde Yerbal en 2013, la cooperativa se ha ido constituyendo en un verdadero eje articulador del trabajo. La cooperativa como institución para la representación se ha transformado ahora en una institución que lleva adelante la organización del trabajo.

\section{Análisis y conclusiones de los procesos de asociatividad en la CRUO}

Al analizar el caso de la CRUO hemos diferenciado fundamentalmente tres etapas (ver Cuadro II). La primera de ellas desde el surgimiento del "fenómeno cartonero" hasta la conformación y consolidación como cooperativa en el año 2008. Luego una segunda etapa, a partir de la puesta en funcionamiento del primer Centro Verde de la cooperativa,Varela, ubicado en el barrio de Villa Soldati y el inicio del proceso de licitación del servicio de recolección de RSU secos. La apertura del Centro Verde Yerbal, junto con la firma de los contratos entre las cooperativas y el GCBA en 2013, indicarían el paso de una segunda a una tercera etapa en la cooperativa.

El traspaso de la primera a la segunda etapa se produce fundamentalmente por la constitución formal como cooperativa. Las disputas entre el GCBA y los recuperadores urbanos junto con las particularidades propias de los grupos que en la actualidad forman la CRUO, derivaron en la formación de la cooperativa. Las primeras formas organizativas se vieron consolidadas con la adquisición de un Centro Verde y con el inicio del proceso de licitación del servicio de gestión de RSU secos por parte del GCBA. La cooperativa comenzó entonces, desde 2008, en esta segunda etapa, a tomar roles muy concretos de representación y de una incipiente administración: canaliza los reclamos de los recuperadores urbanos de la cooperativa, consolida los lazos entre los cooperativistas, estrecha los lazos con las demás cooperativas, negocia con el GCBA, recibe los materiales de los grandes generadores de RSU secos y los comercializa, etc. El paso de la segunda a la tercera etapa está dado por la adquisición del Centro Verde Yerbal y por la firma de los contratos en el 2013. Estos dos factores modificaron el funcionamiento de la cooperativa y produjeron profundas transformaciones en las dinámicas de trabajo. Sin renunciar a su antigua función de representación de los recuperadores urbanos, la CRUO comenzó a forjarse como el eje articulador del trabajo. Desde el 2013 compra el material a los recuperadores 
urbanos, comercializa en grandes cantidades, organiza los recorridos de los grupos de trabajo, brinda un lugar de trabajo, administra incentivos, permite registrar a los trabajadores como monotributistas, acceder a un seguro de accidentes personales, a una obra social, etc.

El eje articulador que ha atravesado desde principios de siglo XXI hasta la actualidad es un proceso de conquistas de derechos que han llevado adelante los recuperadores urbanos de la CABA, en general, y de la CRUO, en particular. Desde la sanción de la Ley 992 en 2002, con distintos ritmos, marchas y contramarchas, los recuperadores urbanos han logrado darle continuidad a un proceso de ampliación de sus derechos. En el mismo proceso (que no podemos pensar como concluido) se han ido reconfigurando sus representaciones y subjetividades, las imágenes sobre ellos mismos. En este proceso no sólo han logrado mejorar sus condiciones de trabajo y de vida de forma considerable, sino que podemos pensar que han forjado una identidad como trabajadores. Algo que quizás antes resultaba impensable. Esta identidad se constituye a partir del reconocimiento de ellos mismos como trabajadores, pero también a partir del reconocimiento de distintos actores sociales fundamentales, como el Estado y diferentes sectores de la sociedad civil, que han modificado sus percepciones en este sentido. Es necesario destacar este proceso de reconfiguración identitaria como un producto de las transformaciones del proceso productivo y de las relaciones establecidas con los distintos actores sociales. Su compleja trama, como destacamos en el trabajo, requiere entonces de un tratamiento en profundidad.

En este período, la identidad de los recuperadores urbanos no ha sido lo único que se ha modificado. La conformación de la cooperativa y las diferentes funciones que esta ha cumplido, junto con las distintas significaciones que ha tomado son, a nuestro entender, los puntos que marcan la principal ruptura entre las distintas etapas identificadas. Tras la sanción de la Ley 1.854 y la conformación como cooperativa y, fundamentalmente, tras la adquisición del Centro Verde Varela y el inicio del proceso de licitación del servicio de recolección de RSU secos, se ha ido conformando una cooperativa que ha cumplido una función fundamentalmente representativa. El rol que toma la cooperativa en esta etapa es, entonces, el de representar los intereses de los recuperadores urbanos, funcionando como una herramienta para mantener e impulsar el proceso de conquista y ampliación de derechos. Por otro lado, la cooperativa funciona desde su inicio como una forma de mantener y consolidar los lazos sociales entre los distintos recuperadores urbanos, logrando coordinar acciones con otras cooperativas o, incluso, estrechar los lazos con grupos de recuperadores urbanos de otras zonas, introduciéndolos a la cooperativa. Tras la firma de los contratos con el GCBA y la adquisición del Centro Verde Yerbal en 2013 la cooperativa ha transformado considerablemente sus funciones. A la representación y la herramienta para el proceso de conquista de derechos ahora es necesario sumarle una función fundamental como articuladora de la actividad productiva, como eje articulador del trabajo. Al mismo tiempo, la propia definición como coope- 
rativa se ha ido transformando y ya no alcanza con una definición clásica para comprender el fenómeno (Paiva, 2004): no sólo su objetivo es recolectar, acopiar y vender material reciclable e interactuar con intermediarios y grandes empresas, ni tampoco lo obtenido se reparte de modo exactamente proporcional. Estas nuevas formas asociativas se conforman como verdaderas instituciones que interactúan con los propios cooperativistas de distintos modos y de acuerdo a diferentes jerarquías. La cooperativa en tanto organizadora del trabajo distribuye funciones y articula la organización productiva, por un lado, y la discusión de las políticas públicas, por el otro.

Con el estudio de este caso hemos logrado dar cuenta de un proceso a través del cual en estos años se ha pasado de una actividad relativamente aislada o individual, mirada con desprecio o rechazo, hacia la formación de nuevas formas asociativas que están constantemente poniendo en juego su significación, los roles a ocupar o las funciones a cumplir. La significación de estas formas asociativas, es decir, el por qué y el para qué se organizan, está constantemente poniéndose en juego entre los propios recuperadores urbanos, en su relación con el Estado local y con la sociedad civil. En el caso estudiado, se han producido distintas respuestas a estas preguntas en momentos diferentes del desarrollo como cooperativa. Si bien no consideramos que resultan formas contrapuestas de asociatividad, creemos que han sido respuestas claramente diferentes producto de las condiciones en las que se han elaborado. Marcar su heterogeneidad resulta, entonces, un primer paso para poder ponerlas en diálogo.

Existe un profundo debate acerca de los procesos de asociatividad en general y, específicamente, sobre el cooperativismo entre los cartoneros. Algunos esbozos de este debate pueden encontrarse en las obras de Schamber (2007), Villanova (2007; 2014) y Maldovan Bonelli (2012; 2014). Lejos de querer cerrar esta discusión, nuestro trabajo, en tanto estudio de caso, pretende constituir un aporte a partir de la reflexión acerca del proceso de conformación de la CRUO.

Las 13 cooperativas que trabajan en la CABA tienen características comunes pero también presentan particularidades, conformando un grupo heterogéneo. Existen pocos trabajos que indaguen en profundidad en las trayectorias de las distintas cooperativas. Creemos que este trabajo puede significar un aporte para un próximo estudio en perspectiva comparativa entre las distintas trayectorias de las cooperativas de la CABA. Para el caso de la CRUO, encontramos en su proceso de formación algunos elementos que pueden resultar relevantes para responder a la pregunta sobre el para qué asociarse o para qué conformar cooperativas.

En primer lugar, la cooperativa se constituyó en un elemento fundamental para comprender el proceso de construcción de una identidad como trabajadores entre los cartoneros. En el propio discurso de los cooperativistas se destacan las transformaciones que realizaron tanto ellos mismos como el resto de los actores de la sociedad, Estado, 
sectores empresarios, etc.

Por otro lado, se ha modificado el lugar que ocupan los cartoneros en el diseño y la implementación de las políticas públicas. La conformación de la cooperativa y la constitución de los vínculos con el GCBA y el Estado local han permitido la discusión del sistema productivo vinculado a la gestión de los residuos de la ciudad. Sin embargo, no debe exagerarse ese aspecto; aclarando que, si bien hoy se pone en discusión y se plantean propuestas para la modificación del sistema, las decisiones siguen tomándose de forma unilateral desde las oficinas del Ministerio de Ambiente y Espacio Público de la CABA. En tercer lugar, con respecto a la condiciones de trabajo, hemos visto que el proceso de formación y consolidación de la cooperativa estuvo mediado por el acceso a mejoras concretas por parte de los cartoneros. Los incentivos han sido un elemento importante en este sentido. Si bien se ha aumentado considerablemente el número de recuperadores urbanos que acceden al mismo, existen análisis que muestran las limitaciones de esta política para igualar las condiciones de los cartoneros con las condiciones de los trabajadores del mercado formal (Villanova, 2014). Por otro lado, la forma de ingreso a la cooperativa por parte de los cartoneros, mediada por el acceso a mejoras concretas, permite poner en cuestión la perspectiva que considera un obstáculo para el cooperativismo la falta de "conciencia" o "educación asociativa” entre los recuperadores urbanos (Escliar et al., 2007).

Por último, la formación de la cooperativa permitiría el acceso a mercados que anteriormente los cartoneros tenían negados. Previo a la formación de cooperativas, los cartoneros estaban obligados a vender el material individualmente a los distintos intermediarios. La organización en la venta del material permitiría vender a nivel industrial, "saltando" los intermediarios y logrando apropiarse de esos ingresos por la comercialización. Ciertas lecturas apuntan en ese sentido (Escliar et al., 2007). Sin embargo, es necesario señalar que en el caso de la CRUO se han presentado obstáculos para el acceso al nivel industrial. Es quizás en este aspecto donde se hace más notoria la necesidad de la intervención del Estado en el mercado.

Todos estos aspectos dan cuenta del proceso de formación de la CRUO y, al mismo tiempo, cada uno de ellos pone en evidencia la importancia que juega el rol del Estado, sin el cual es imposible pensar el proceso de conformación y consolidación de la cooperativa. Es necesario, entonces, lograr poner en discusión las distintas aristas en las que la intervención del Estado resulta perentoria para consolidar la posición de las cooperativas en el sistema productivo vinculado a la gestión de los residuos de la ciudad. 


\section{Anexo: Cuadros y mapas}

\section{Cuadro I}

Hitos relevantes en el proceso de transformación del sistema de recolección de residuos en la CABA

\begin{tabular}{|c|c|c|}
\hline \multicolumn{3}{|c|}{$\begin{array}{l}\text { Principales hitos en el proceso de transformación del sistema de recolección } \\
\text { de residuos en la CABA }\end{array}$} \\
\hline 1976 & Creación del CEAMSE & $\begin{array}{l}\text { Artículo } 6^{\circ} \text { de la Ordenanza } N^{\circ} 33.581 \\
\text { prohíbe el "cirujeo" } \\
\text { Aplicación del Método de Relleno } \\
\text { Sanitario }\end{array}$ \\
\hline $\begin{array}{l}\text { Diciembre } \\
2002\end{array}$ & Ley $992 / 02$ & $\begin{array}{l}\text { Deroga el Art. } 6^{\circ} \text { de la Ordenanza } \\
\mathrm{N}^{\circ} 33.581 \\
\text { Deja sin efecto el entierro } \\
\text { indiscriminado como disposición } \\
\text { final de los residuos } \\
\text { Incorpora a los recuperadores urbanos } \\
\text { como parte del servicio de residuos } \\
\text { reciclables de higiene urbana } \\
\text { Crea el Registro Único Obligatorio } \\
\text { Permanente de Recuperadores de } \\
\text { Materiales Reciclables (RUR) }\end{array}$ \\
\hline Mayo 2003 & Decreto 662/03 & $\begin{array}{l}\text { Crea el Programa de Recuperadores } \\
\text { Urbanos y Reciclado de RSU (PRU) }\end{array}$ \\
\hline $\begin{array}{l}\text { Noviembre } \\
2005\end{array}$ & Ley “de Basura Cero” (1.854/05) & \\
\hline Julio 2007 & $\begin{array}{l}\text { Suspensión de los trenes cartoneros } \\
\text { de TBA }\end{array}$ & \\
\hline Julio 2008 & $\begin{array}{l}\text { Implementación de un Nuevo Sistema } \\
\text { de Recolección de Residuos }\end{array}$ & $\begin{array}{l}\text { División entre residuos húmedos } \\
\text { y residuos secos }\end{array}$ \\
\hline Abril 2010 & $\begin{array}{l}\text { Presentación de los pliegos de } \\
\text { licitación pública de los servicios } \\
\left.\text { (Expediente } N^{o} 350.165 / 2010\right)\end{array}$ & \\
\hline Marzo 2011 & $\begin{array}{l}\text { Firma de contratos entre el GCBA, } \\
\text { empresas privadas y las cooperativas } \\
\text { para la prestación de los servicios } \\
\text { de recolección }\end{array}$ & \\
\hline Enero 2013 & $\begin{array}{l}\text { Nueva firma entre el GCBA } \\
\text { y } 13 \text { cooperativas }\end{array}$ & \\
\hline
\end{tabular}




\section{Cuadro II}

Transformación de la función de la cooperativa a lo largo del tiempo

\begin{tabular}{|l|l|l|l|l|}
\hline \multicolumn{2}{|l|}{ Cooperativa como institución para la representación } & \multicolumn{2}{l|}{ Cooperativa como organizadora del trabajo } \\
\hline 2000-2008: Primera Etapa & 2008-2013: Segunda Etapa & 2013-2015: Tercera Etapa \\
\hline 2000 - 2008 & 2008 & 2013 & 2015 \\
\hline $\begin{array}{l}\text { Organización en torno al } \\
\text { tren cartonero }\end{array}$ & $\begin{array}{l}\text { Comienzo de trabajo en } \\
\text { el Centro Verde Varela }\end{array}$ & $\begin{array}{l}\text { Comienzo de trabajo en } \\
\text { el Centro Verde Yerbal }\end{array}$ & \\
\hline
\end{tabular}

\section{Mapa I}

\section{Trayecto Ferrocarril Línea Sarmiento Moreno-Once y ubicación}
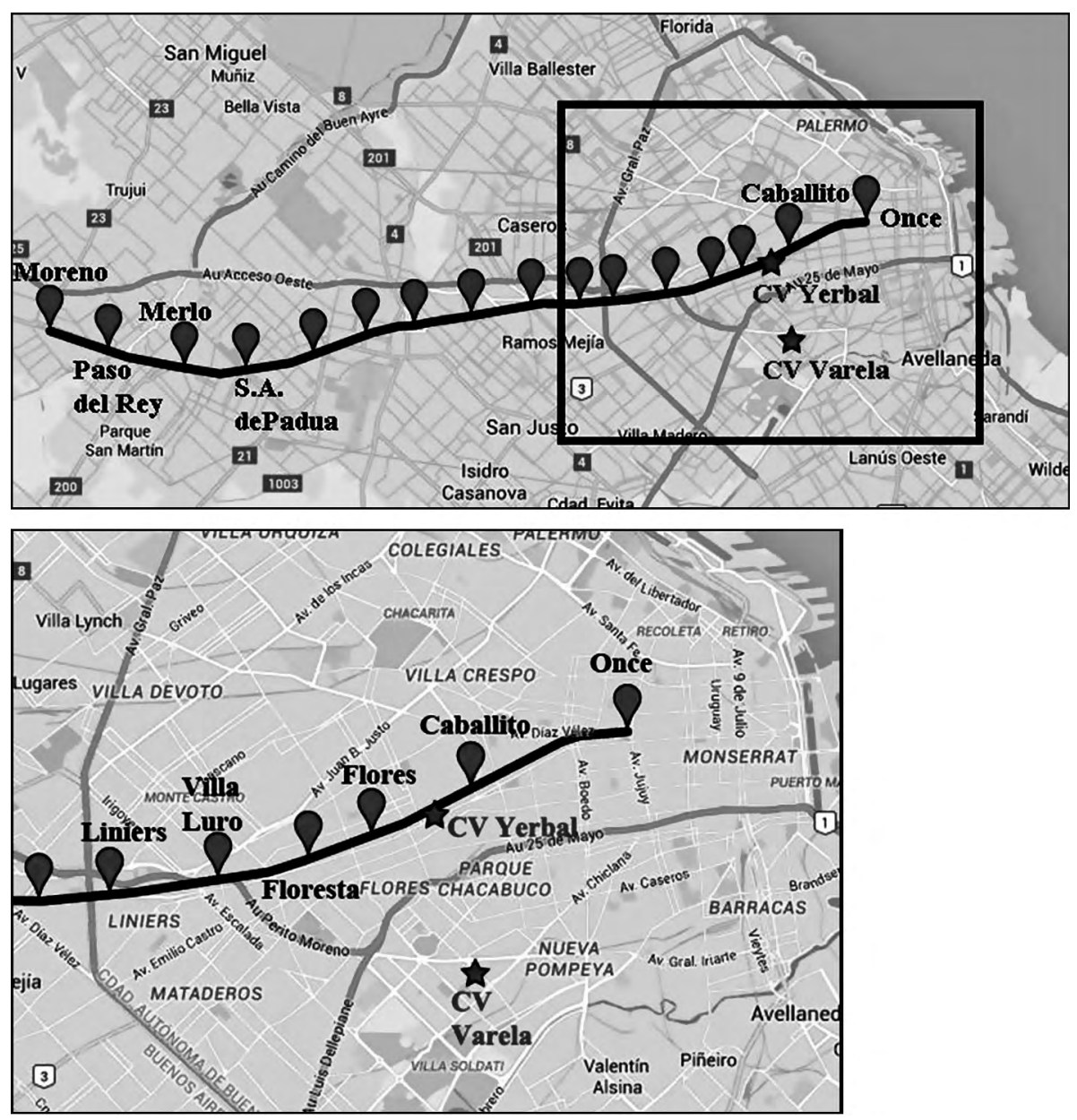


\begin{abstract}
${ }^{1}$ Trabajo desarrollado en el marco del Proyecto de Investigación UNLa No 181 (2013-2015).
${ }^{2}$ La principal cooperativa de recuperadores urbanos de la CABA, en lo que a número de trabajadores respecta, es el Movimiento de Trabajadores Excluidos (MTE), donde se organizan más de tres mil trabajadores.

${ }^{3}$ Durante varios meses realizamos trabajo de campo a través de observación no participante y entrevistas semi-estructuradas con distintos actores ligados a la cooperativa: trabajadores en distintos cargos (presidente, delegados, coordinadores y socios), trabajadores externos contratados por la cooperativa y trabajadores del Estado local, entre otros. El análisis realizado en este artículo es el resultado de la sistematización de nuestras primeras aproximaciones en el trabajo de campo.

${ }^{4}$ Concebimos las políticas públicas como el "conjunto de acciones y omisiones que manifiestan una determinada modalidad de intervención del Estado en relación con una cuestión que concita la atención, interés o movilización de otros actores en la sociedad civil. De dicha intervención puede inferirse una cierta direccionalidad, una determinada orientación normativa, que previsiblemente afectará el futuro curso del proceso social hasta entonces desarrollado en torno a la cuestión" (Oszlak y O’Donnell, 1995: 112).

${ }^{5}$ La ley $\mathrm{N}^{\circ}$ 992/02 se encuentra disponible en: http://www.buenosaires.gob.ar/areas/leg_tecnica/sin/normapop09.php? $\mathrm{id}=31157 \& \mathrm{qu}=\mathrm{c} \& \mathrm{ft}=0 \& \mathrm{cp}=\& \mathrm{rl}=1 \& \mathrm{rf}=0 \& \mathrm{im}=\& \mathrm{ui}=0 \&$ printi $=\&$ pelikan $=1 \&$ sezion $=796791$ \&primera $=0 \&$ mot_toda $=\&$ mot_frase $=\&$ mot_alguna $=$

${ }_{6}^{6}$ El decreto $\mathrm{N}^{\circ}$ 622/03 está disponible en: http://www.buenosaires.gob.ar/areas/leg_tecnica/sin/normapop09.php? $\mathrm{id}=37081 \& \mathrm{qu}=\mathrm{c} \& \mathrm{ft}=0 \& \mathrm{cp}=\& \mathrm{rl}=1 \& \mathrm{rf}=0 \& \mathrm{im}=\& \mathrm{xi}=0 \&$ printi $=\&$ pelikan $=1 \&$ sezion $=796791 \&$ primera $=0 \&$ mot_toda $=\&$ mot_frase $=\&$ mot_alguna $=$

7 La ley 1.854 está disponible en: http://www.buenosaires.gob.ar/areas/leg_tecnica/sin/normapop09.php? $\mathrm{d}=81508 \& \mathrm{qu}=\mathrm{c} \& \mathrm{ft}=0 \& \mathrm{cp}=\& \mathrm{rl}=1 \& \mathrm{rf}=0 \& \mathrm{im}=\& u i=0 \& p$ rinti $=\&$ pelikan $=1 \&$ sezion $=796791 \& \mathrm{primera}=0 \&$ mot_toda $=\&$ mot_frase $=\&$ mot_alguna $=$
\end{abstract}

${ }^{8}$ Utilizamos el término "grandes generadores" para aquellos que están comprendidos en la ley 1.854/05 como "generadores especiales". En su artículo No 12 se clasifica a los generadores de residuos sólidos urbanos en "individuales" y "especiales". Según el artículo N $N^{\circ} 13$ los generadores especiales son aquellos "que pertenecen a los sectores comerciales, institucionales e industriales que producen residuos sólidos urbanos en una cantidad, calidad o en condiciones tales que, a juicio de la autoridad de aplicación, requieran de la implementación de programas específicos de gestión, previamente aprobados por la misma".

${ }^{9}$ Denominamos "Centros Verdes" a los centros de selección del material que, a partir de la ley 1.854/05 debían ser asignados a las distintas cooperativas. Como veremos más adelante el primero de estos Centros Verdes se pondrá en funcionamiento en el año 2007.

${ }^{10}$ Se denominan "trenes cartoneros" a aquellos servicios especiales de transporte ferroviario que se pusieron en funcionamiento a partir de la crisis del 2001 y el considerable aumento de pasajeros que se trasladaban con sus carros desde el GBA hacia la CABA para “cartonear”. El servicio era proporcionado por las empresas TBA (Línea Sarmiento y Línea Mitre) y Metropolitano (Línea San Martín y Línea Roca).Ver, para el caso, la nota "Dejó de circular el tren blanco de los cartoneros del Sarmiento", Clarín, 06/07/2007. Disponible en: http:// edant.clarin.com/diario/2007/07/06/laciudad/h-04201.htm

${ }^{11}$ Ver nota "Un lugar en el mundo para los cartoneros", Página/12, 30/03/2011. Disponible en: http://www. pagina12.com.ar/diario/sociedad/3-165183-2011-03-30.html

12 Ver nota "Contrato para los cartoneros", Página/12, 11/01/2013. Disponible en: http://www.pagina12. com.ar/diario/sociedad/3-211617-2013-01-11.htm Las 13 cooperativas que firmaron los contratos con el GCBA son: El Amanecer de los Cartoneros (MTE), Recuperadores Urbanos del Oeste, El Ceibo, El Álamo, Las Madreselvas, Alelí, Cooperativa del Oeste, Reciclando Sueños, Reciclando Trabajo y Dignidad, Baires Cero Con, El Trébol, Cartonera del Sur y Primavera. Todos los contratos firmados en enero del 2013 se encuentran disponibles en: http://www.residuosurbanos.org.an

${ }^{13}$ Otros trabajos indagan en la relevancia que tiene el tren en la organización de los trabajadores e introduce como interesante variable para el análisis la cuestión territorial: el barrio (Gorbán, 2006). Precisamente, queda aquí planteada, entonces, la necesidad de rastrear los modos específicos en que se articulan en la CRUO la cuestión territorial, es decir, la vida en los barrios (principalmente al oeste del GBA pero también los barrios de Once y Chacarita) con la organización del trabajo al interior de la cooperativa.

${ }^{14}$ Según integrantes de la CRUO, a fines de 2015 el monto del subsidio ha ido creciendo con el tiempo, comenzando con $\$ 200$ en 2009 , se ubicó en 2012 en $\$ 1.600$, en 2014 en $\$ 2.000$ y a principios de 2015 en $\$ 2.700$. ${ }^{15}$ La relación entre agentes sindicales de ATE y la cooperativa es una relación compleja que bordea los canales institucionales más tradicionales. Para ahondar en ella, resulta interesante consultar la obra de Johanna Maldovan Bonelli (Maldovan Bonelli, 2014). 


\begin{abstract}
${ }^{16}$ Según integrantes de la cooperativa y trabajadores del Estado local, al momento de iniciar la política de otorgamiento de incentivos, el GCBA implementó una serie de cupos. Estos se reparten en distintas proporciones a las diferentes cooperativas de la ciudad. Las cooperativas son las encargadas de decidir quiénes ocupan esos cupos y perciben el incentivo mensual. Luego, cada cooperativa conforma una lista de espera donde incluye a todos sus asociados que aún no perciben el ingreso. Por otro lado, el GCBA pide como requisitos para percibir el incentivo cumplir con ciertas reglas como son, por ejemplo, la asistencia laboral.

${ }^{17}$ La Cooperativa Ecológica de Recicladores del Bajo Flores (CERBAF) tuvo una larga trayectoria que se remonta a la década de 1990. En el 2007 logró quedar a cargo del primer Centro Verde de CABA, ubicado en la avenida Varela 2505 (esquina Janer), en el barrio deVilla Soldati. Luego de que la cooperativa haya dejado de funcionar, se inició el proceso de disputa por la gestión del Centro Verde allí ubicado (Maldovan Bonelli, 2014: 148). ${ }^{18}$ Según testimonios de propios integrantes de la cooperativa y de empleados del Estado local, que trabajan allí, la cooperativa comenzó con un grupo histórico que comenzó a organizarse en los trenes cartoneros, conformado por aproximadamente 500 personas, llegando a contar en la actualidad con más de 1500 trabajadores. ${ }^{19}$ Uno de los principales obstáculos destacados por integrantes de la CRUO han sido las largas esperas para ingresar en las industrias y concretar la venta. "Nosotros no podemos tener un camión parado por uno o dos días" decía Andrés Vázquez, encargado de la comercialización en la cooperativa. En definitiva, el núcleo de la cuestión sigue siendo la relativa poca cantidad de material que maneja la cooperativa en relación con las cantidades que se manejan a nivel industrial.

${ }^{20}$ En diversas entrevistas, distintos actores destacaron la diferencia de precios que ofrece la CRUO respecto a los intermediarios que compran el material en GBA. Andrés Vázquez, a cargo de la comercialización en la cooperativa, nos informó acerca de esta diferencia porcentual.Aquí se pone en evidencia la necesidad de investigaciones sobre el sector de intermediarios y galponeros, que indaguen específicamente en el impacto que tuvieron las transformaciones de las últimas décadas en el proceso de recolección y comercialización de RSU secos.
\end{abstract}




\section{Referencias bibliográficas}

Dimarco, S. (2007) “¿Podremos mirar más allá de la basura? Raneros, cirujas y cartoneros: historias detrás de la basura”. Papeles del CEIC, Vol. 2007/2, septiembre. Disponible en: http://www.identidadcolectiva.es/pdf/33.pdf (Acceso el 21 de marzo de 2016).

Escliar, V., Mutuberría Lazarini, V., Rodríguez, M.F. y Rodríguez, P. (2007) "Cartoneros: ¿Una práctica individual o asociativa? Ciudad de Buenos Aires, año 2004-2005”. Cuadernos del CCC, Cuaderno de Trabajo No 75. Disponible en: http://www.centrocultural.coop/descargas/cuadernos-del-ccc/cartoneros-una-practica-individual-o-asociativaciudad-de-buenos-aires-anio-2004---2005.htm (Acceso el 09 de enero de 2016).

Fajn, J.G. (2002) "Exclusión social y autegestión"Cooperativas de recicladores de residuos". Revista Idelcoop, Vol 29, N 139, pp. 164-193. Disponible en: http://www.idelcoop.org.ar/sites/default/files/revista/articulos/pdf/2002_261327532.pdf (Acceso el 21 de marzo de 2016).

Gorbán, D. (2006) “Trabajo y cotidianeidad. El barrio como espacio de trabajo de los cartoneros del Tren Blanco". Trabajo y Sociedad, N 8:67-91. Disponible en: http:// www.unse.edu.ar/trabajoysociedad/Gorban.pdf (Acceso el 21 de marzo de 2016).

Maldovan Bonelli, J. (2012) "Trabajo, asociatividad y acción colectiva: el caso de las cooperativas de recuperadores urbanos". Trabajo y Sociedad, No 19:117-131. Disponible en: http://www.redalyc.org/articulo.oa?id=387334691007 (Acceso el 09 de enero de 2016).

Maldovan Bonelli, J. (2014) Del trabajo autónomo a la autonomía de las organizaciones. La construcción de asociatividad en las cooperativas de recuperadores urbanos de la Ciudad de Buenos Aires 2007-2012 (Tesis doctoral no publicada), Facultad de Ciencias Sociales, Universidad de Buenos Aires, Buenos Aires.

Oszlak O. y O`Donnell G. (1995) "Estado y Políticas estatales en América Latina: hacia una estrategia de investigación”. Redes, Vol. 2, N 4:128. Disponible en: http:// www.redalyc.org/articulo.oa?id=90711285004 (Acceso el 20 de marzo de 2016)

Paiva, V. (2004) "Las cooperativas de recuperadores y la gestión de residuos sólidos urbanos en el Área Metropolitana de Buenos Aires, 2003". Theomai, N 99. Disponible en: http://www.redalyc.org/pdf/124/12499309.pdf (Acceso el 21 de marzo de 2016). 
Paiva, V. (2005) "Modos formales e informales de recolección y tratamiento de residuos, Buenos Aires, siglos XVI al XX”. Crítica, No 150, noviembre. Instituto de Arte Americano e Investigaciones Estética, Facultad de Arquitectura, Diseño y Urbanismo (FADU), Universidad de Buenos Aires. Disponible en: http://www.iaa.fadu.uba.ar/publicaciones/critica/0150.pdf (Acceso el 09 de enero de 2016).

Paiva, V. (2013) “Cartoneros, recolección informal, ambiente y políticas públicas en Buenos Aires 2001-2012”. urbe. Revista Brasileira de Gestãd Urbana (Brazilian Journal of Urban Management),Vol. 5, No 1:149-158. Disponible en: http://www.redalyc.org/articulo.oa?id=193128451011 (Acceso el 09 de enero de 2016).

Perelman, M. (2008) "De la vida en la Quema al Trabajo en las calles. El cirujeo Ciudad de Buenos Aires". Avá. Revista de Antropología, No 12:117-135. Disponible en: http:// www.redalyc.org/pdf/1690/169013839007.pdf (Acceso el 09 de enero de 2016).

Perelman, M. y Boy, M. (2010) "Cartoneros en Buenos Aires: nuevas modalidades de encuentro". Revista Mexicana de Sociología, 72 No 3:393-418. Disponible en: http:// www.scielo.org.mx/scielo.php?script $=$ sci_arttext\&pid=S0188-25032010000300002 (Acceso el 09 de enero de 2016).

Schamber, P. (2007) "Modalidades organizativas de los cartoneros". En Schamber, P. y Suárez, F. (comp.) Recicloscopio, Buenos Aires: Prometeo Libros/Universidad Nacional de General Sarmiento, pp.217-220.

Schamber P. (2012) "Proceso de integración de los cartoneros de la Ciudad Autónoma de Buenos Aires. Del reconocimiento a la gestión de Centros Verdes y la recolección selectiva", en Documentos de Trabajo de WIEGO, No 24, Abril. Disponible en: http:// wiego.org/683A2B72-F854-4619-800D-B93C3941C667/FinalDownload/DownloadId-A1AC3A7935E103544DAC31FB8DC77643/683A2B72-F854-4619-800DB93C3941C667/sites/wiego.org/files/publications/files/Schamber_WIEGO_WP24. pdf ISBN 978-82-95095-24-3 (Acceso 13 de enero de 2016).

Schamber, P. y Suárez, F. (2002) “Cirujeo y gestión de residuos. Una mirada sobre el circuito informal del reciclaje en el conurbano bonaerense”. Realidad Económica 190 www.iade.org.ar/modules/noticias/article.php?storyid=703.

Schamber, P. y Suárez, F. (2012) “Logros y desafíos a diez años del reconocimiento de los cartoneros en la CABA (2002-2012)”. Realidad Económica 271:102-132. Disponible en: http://www.iade.org.ar/modules/noticias/article.php?storyid=4266 (Acceso el 09 de enero de 2016). 
Vergara, G. (2009) "Conflicto y emociones. Un retrato de la vergüenza en Simmel, Elías y Giddens como excusa para interpretar prácticas en contextos de expulsión”. En Scribano, A. y Figari, C. Cuerpos, subjetividades y conflictos: hacia una sociología de los cuerpos $y$ las emociones desde Latinoamérica. Buenos Aires: CLACSO y CICCUS, 35-52.

Villanova, N. (2008) "Dignamente explotados". Revista Razón y Revolución, N 18:221-226. Disponible en: http://revistaryr.org.ar/index.php/RyR/article/view/112 (Acceso el 09 de enero de 2016).

Villanova, N. (2012) "Cartoneros y piqueteros. La lucha de los recuperadores urbanos, el Tren Blanco y el Argentinazo. Ciudad Autónoma de Buenos Aires, 1999-2011”. Revista Razón y Revolución 23:97-111. Disponible en: http://www.revistaryr.org.ar/index. $\mathrm{php/RyR/article/view/21} \mathrm{(Acceso} \mathrm{el} 09$ de enero de 2016).

Villanova, N. (2014) "Los cartoneros y la estatización de su condición como población sobrante para el capital por intermedio de las cooperativas. Ciudad de Buenos Aires, 2001-2012". Trabajo y Sociedad 23: 67-91. Disponible en: http://www.redalyc. org/pdf/3873/387334695004.pdf (Acceso el 09 de enero de 2016). 